\title{
A converse theorem for second-order modular forms of level $N$
}

\author{
by \\ Özlem Imamō̄lu (Zürich) and Yves Martin (Santiago)
}

1. Introduction. The Eisenstein series twisted by modular symbols is the prime example of a second-order automorphic form. It was introduced by Goldfeld in [6] to study the distribution of modular symbols and to provide a new approach to Szpiro's conjecture. Since then, these series have been studied and generalized by many authors ([2], [4], [7], [8], [11]-[13]). The twisted Eisenstein series is not an automorphic form in the classical sense but satisfies a shifted automorphy relation which involves the ordinary Eisenstein series.

Automorphic functions of the same kind were also encountered by Kleban and Zagier in their research on crossing probabilities. In [9] they initiated the study of $n$th order modular forms, i.e. functions whose deviation from modularity is an $(n-1)$ th order modular form.

In this paper we will restrict ourselves to the study of second order modular forms. Fix positive integers $N$ and $k$ with $k$ even. Let $\mathcal{H}$ be the complex upper half-plane. If $f=f(\tau): \mathcal{H} \rightarrow \mathbb{C}$ is any function and $\gamma=$ $\left(\begin{array}{ll}a & b \\ c & d\end{array}\right)$ a matrix in $\mathrm{GL}_{2}^{+}(\mathbb{R})$, the usual slash operator is

$$
\left.f(\tau)\right|_{k}[\gamma]=(\operatorname{det} \gamma)^{k / 2}(c \tau+d)^{-k} f\left(\frac{a \tau+b}{c \tau+d}\right) .
$$

By linearity this defines an action of the group ring $\mathbb{Z} \mathrm{GL}_{2}^{+}(\mathbb{R})$ on the set of holomorphic functions on $\mathcal{H}$.

A second-order modular form $F(\tau)$ of weight $k$ and level $N$ is a function $F: \mathcal{H} \rightarrow \mathbb{C}$ such that

(i) $F(\tau)$ is holomorphic on $\mathcal{H}$,

(ii) $\left.F(\tau)\right|_{k}[\gamma]$ has at most polynomial growth at the cusps for each $\gamma \in$ $\Gamma_{0}(N)$, 
(iii) $\left.F(\tau)\right|_{k}\left[\left(\gamma_{1}-I_{2}\right)\left(\gamma_{2}-I_{2}\right)\right]=0$ for all $\gamma_{1}, \gamma_{2}$ in $\Gamma_{0}(N)$,

(iv) $\left.F(\tau)\right|_{k}\left[\gamma-I_{2}\right]=0$ for every parabolic element $\gamma$ in $\Gamma_{0}(N)$.

The $\mathbb{C}$-vector space of second-order modular forms of weight $k$ and level $N$ is denoted by $\mathfrak{M}_{k}^{2}(N)$. It has been investigated in [1] and [5]. In particular, a decomposition theorem in terms of elliptic modular forms is proved in [1], while in [5] the dimension of the space is found and a cohomological interpretation is given. Denote by $\mathfrak{M}_{k}^{1}(\Gamma)$ the space of elliptic modular forms of weight $k$ over a group $\Gamma \subset \mathrm{SL}_{2}(\mathbb{R})$, and by $\mathfrak{M}_{k}^{1}(N)$ the same space whenever $\Gamma=\Gamma_{0}(N)$. Clearly $\mathfrak{M}_{k}^{1}(N) \subseteq \mathfrak{M}_{k}^{2}(N)$. In most cases the containment is proper.

By (i), (ii) and (iv) above, any second-order modular form has a Fourier series representation and hence an obvious Dirichlet series associated to it. In this note we investigate some properties of the latter. More precisely, for a Fourier series $F(\tau)=\sum_{n=0}^{\infty} A_{n} \exp (2 \pi i n \tau)$ and for $s$ in $\mathbb{C}$ let

$$
L(F ; s)=\sum_{n=1}^{\infty} A_{n} n^{-s} \quad \text { and } \quad \Lambda_{N}(F ; s)=\left(\frac{2 \pi}{\sqrt{N}}\right)^{-s} \Gamma(s) L(F ; s) .
$$

For any primitive Dirichlet character $\chi$ of conductor $m$ with $\operatorname{gcd}(N, m)=1$ let

$$
L\left(F_{\chi} ; s\right)=\sum_{n=1}^{\infty} \chi(n) A_{n} n^{-s} \quad \text { and } \quad \Lambda_{N m^{2}}\left(F_{\chi} ; s\right)=\left(\frac{2 \pi}{m \sqrt{N}}\right)^{-s} \Gamma(s) L\left(F_{\chi} ; s\right) .
$$

(The corresponding definition for the trivial character mod $m$ is slightly different and is given in Section 4.) Our main result is a converse theorem for $\mathfrak{M}_{k}^{2}(N)$.

THEOREM. Let $\left\{A_{n}\right\}_{n}$ and $\left\{B_{n}\right\}_{n}$ be two sequences in $\mathbb{C}$ with $A_{n}=$ $O\left(n^{\nu}\right)$ and $B_{n}=O\left(n^{\nu}\right)$ for some $\nu>0$. Define functions

$$
F(\tau)=\sum_{n=0}^{\infty} A_{n} \exp (2 \pi i n \tau) \quad \text { and } \quad G(\tau)=\sum_{n=0}^{\infty} B_{n} \exp (2 \pi i n \tau)
$$

on $\mathcal{H}$. Then statements $(\mathrm{A})$ and $(\mathrm{B})$ below are equivalent.

(A) $F(\tau)$ and $G(\tau)$ are in $\mathfrak{M}_{k}^{2}(N)$ with $G(\tau)=\left.F(\tau)\right|_{k}[w(N)]$.

(B) The following conditions on the corresponding Dirichlet series hold.

The series

$$
\Lambda_{N}(F ; s)+\frac{A_{0}}{s}+i^{k} \frac{B_{0}}{k-s}
$$

has a holomorphic continuation to the whole s-plane, is bounded on any vertical strip and satisfies

$$
\Lambda_{N}(F ; s)=i^{k} \Lambda_{N}(G ; k-s) .
$$


If $a_{0}^{\bar{\chi}}\left(r e s p . b_{0}^{\chi}\right)$ is the constant term in the Fourier series of $\left.G_{\chi}(\tau)\right|_{k}\left[w\left(N p^{2}\right)\right]\left(\right.$ resp. $\left.\left.F_{\bar{\chi}}(\tau)\right|_{k}\left[w\left(N p^{2}\right)\right]\right)$, where $\chi$ is any Dirichlet character $\bmod p$ and $p$ is an odd prime with $\operatorname{gcd}(N, p)=1$, then:

(i) The function

$$
i^{k} \Lambda_{N p^{2}}\left(G_{\chi} ; k-s\right)-C_{\chi} \Lambda_{N p^{2}}\left(F_{\bar{\chi}} ; s\right)+\frac{a_{0}^{\bar{\chi}}}{s}-i^{k} C_{\chi} \frac{b_{0}^{\chi}}{k-s}
$$

has a holomorphic continuation to the whole s-plane, bounded on any vertical strip.

(ii) The function $i^{k} \Lambda_{N p^{2}}\left(G_{\chi} ; k-s\right)-C_{\chi} \Lambda_{N p^{2}}\left(F_{\bar{\chi}} ; s\right)$ is the (completed) L-function of a modular form in $\mathfrak{M}_{k}^{1}\left(\Gamma_{0}\left(N p^{2}\right) \cap \Gamma_{1}(p)\right)$.

(iii) The Dirichlet series $\sum_{\chi} \chi(u) C_{\bar{\chi}} W(\chi) \Lambda_{N p^{2}}\left(G_{\chi} ; s\right)$ (where the sum is over all Dirichlet characters mod p) has a holomorphic continuation to the whole s-plane, is bounded on vertical strips and satisfies

$$
\sum_{\chi} \chi(u) C_{\bar{\chi}} W(\chi)\left\{i^{k} \Lambda_{N p^{2}}\left(G_{\chi} ; k-s\right)-C_{\chi} \Lambda_{N p^{2}}\left(F_{\bar{\chi}} ; s\right)\right\}=0,
$$

whenever $u \in \mathbb{Z}$ is such that $(p \pm 1)^{2} \equiv 0(\bmod u N)$.

Here and from now on, $w(x)=\left(\begin{array}{cc}0 & -1 \\ x & 0\end{array}\right)$ in $\mathrm{GL}_{2}^{+}(\mathbb{R}), W(\chi)$ is the Gauss sum associated to $\chi$ and $C_{\chi}=\chi(-N) W(\chi) W(\bar{\chi})^{-1}$.

Weil's converse theorem allows us to replace condition (ii) above by a set of functional equations. In Section 5 we rephrase our theorem in those terms (see Theorem 2).

After the completion of this work we learned about a preprint of Diamantis, Knopp, Mason and O'Sullivan [3]. They study a similar Dirichlet series and prove a "partial" converse theorem (Thm. 14 in [3]). Their result differs from ours in two ways. First, the space of forms considered in [3] is not the one studied in this paper. Secondly, in the converse direction they impose a "strong periodicity relation" which we do not assume here.

This article is organized as follows. In the next section we recall Weil's converse theorem in the form that we need. In Section 3 we give the definition of a second-order modular form. Then, in the following section we give an equivalent description of those second-order modular forms using their Fourier series and Dirichlet characters. Finally, in Section 5 we state and prove the converse theorem of the title.

Notation. For convenience we use the symbols $I_{2}$ and $\theta(x)$ for, respectively, the matrices $\left(\begin{array}{ll}1 & 0 \\ 0 & 1\end{array}\right)$ and $\left(\begin{array}{ll}1 & x \\ 0 & 1\end{array}\right)$ in $\mathrm{GL}_{2}^{+}(\mathbb{R})$. Also, we write $e(x)$ for $\exp (2 \pi i x)$. 
2. Weil's converse theorem. In this section we recall Weil's converse theorem and some standard lemmas which we will need. For their proofs see for example [10, p. 117].

Lemma 1. Let $f: \mathcal{H} \rightarrow \mathbb{C}$ be a holomorphic function with a Fourier series representation $f(\tau)=\sum_{n=0}^{\infty} a_{n} e(n \tau)$ which converges absolutely and uniformly on compact subsets of $\mathcal{H}$. If there is a real number $\nu>0$ such that $f(\tau)=O\left(\operatorname{Im}(\tau)^{-\nu}\right)$ as $\operatorname{Im}(\tau) \rightarrow 0$ uniformly in $\operatorname{Re}(\tau)$, then $a_{n}=O\left(n^{\nu}\right)$.

LEMMA 2. Let $\left\{a_{n}\right\}_{n=0}^{\infty}$ be a sequence of complex numbers and set

$$
f(\tau)=\sum_{n=0}^{\infty} a_{n} e(n \tau) \quad \text { for } \tau \in \mathcal{H}
$$

If $a_{n}=O\left(n^{\nu}\right)$ for some real number $\nu>0$, then the series (1) converges absolutely and uniformly on compact subsets of $\mathcal{H}$. In particular $f$ is a holomorphic function on $\mathcal{H}$. Moreover both $f(\tau)=O\left(\operatorname{Im}(\tau)^{-\nu-1}\right)$ as $\operatorname{Im}(\tau) \rightarrow 0$ and $f(\tau)-a_{0}=O\left(e^{-2 \pi \operatorname{Im}(\tau)}\right)$ as $\operatorname{Im}(\tau) \rightarrow \infty$, uniformly in $\operatorname{Re}(\tau)$.

By these results, the holomorphic functions on $\mathcal{H}$ satisfying the hypothesis of Lemma 1 correspond bijectively to the sequences $\left\{a_{n}\right\}_{n}$ in $\mathbb{C}$ with $a_{n}=O\left(n^{\nu}\right)$ for some $\nu>0$.

Definition 1. Let $f(\tau)=\sum_{n=0}^{\infty} a_{n} e(n \tau)$ be a function as in Lemma 1. For $s$ in $\mathbb{C}$ define

$$
L(f ; s)=\sum_{n=1}^{\infty} a_{n} n^{-s} \text { and } \quad \Lambda_{N}(f ; s)=\left(\frac{2 \pi}{\sqrt{N}}\right)^{-s} \Gamma(s) L(f ; s) .
$$

Let $\chi$ be a primitive Dirichlet character of conductor $m$ with $\operatorname{gcd}(N, m)=1$. For $s$ in $\mathbb{C}$ define

$L(f, \chi ; s)=\sum_{n=1}^{\infty} \chi(n) a_{n} n^{-s}$ and $\Lambda_{N m^{2}}(f, \chi ; s)=\left(\frac{2 \pi}{m \sqrt{N}}\right)^{-s} \Gamma(s) L(f, \chi ; s)$.

Since $a_{n}=O\left(n^{\nu}\right)$ for some $\nu>0$, the series $L(f ; s), L(f, \chi ; s), \Lambda_{N}(f ; s)$ and $\Lambda_{N m^{2}}(f, \chi ; s)$ are absolutely and uniformly convergent on compact subsets of the half-plane $\operatorname{Re}(s)>\nu+1$.

The following result is due to Hecke.

Proposition 1. Let $f(\tau)=\sum_{n=0}^{\infty} a_{n} e(n \tau)$ and $g(\tau)=\sum_{n=0}^{\infty} b_{n} e(n \tau)$ be functions on $\mathcal{H}$ satisfying the hypothesis of Lemma 1 . Then statements (A) and (B) below are equivalent.

(A) $g(\tau)=\left.f(\tau)\right|_{k}[w(N)]$

(B) $\Lambda_{N}(f ; s)$ and $\Lambda_{N}(g ; s)$ admit meromorphic continuations to the whole s-plane and they satisfy

$$
\Lambda_{N}(f ; s)=i^{k} \Lambda_{N}(g ; k-s) .
$$


Moreover $\Lambda_{N}(f ; s)+a_{0} / s+i^{k} b_{0} /(k-s)$ is holomorphic on the whole $s$-plane and bounded on any vertical strip.

Hecke used Proposition 1 to get his converse theorem for elliptic modular forms over $\mathrm{SL}_{2}(\mathbb{Z})$. André Weil [15] proved such a converse theorem for modular forms over congruence subgroups $\Gamma_{0}(N)$ by considering Fourier series twisted with Dirichlet characters.

Definition 2. Let $f: \mathcal{H} \rightarrow \mathbb{C}$ be a holomorphic function represented by a Fourier series $f(\tau)=\sum_{n=0}^{\infty} a_{n} e(n \tau)$, and $\chi$ any primitive Dirichlet character of conductor $m$. The Fourier series twisted by $\chi$ is $f_{\chi}(\tau)=$ $\sum_{n=0}^{\infty} \chi(n) a_{n} e(n \tau)$.

Notice that $\Lambda_{N m^{2}}\left(f_{\chi} ; s\right)=\Lambda_{N m^{2}}(f, \chi ; s)$. A standard computation yields

$$
\left.\sum_{u(\bmod m)} \bar{\chi}(u) f(\tau)\right|_{k}[\theta(u / m)]=W(\bar{\chi}) f_{\chi}(\tau) .
$$

Next, we recall Weil's converse theorem in a form that is convenient for our purposes.

Let $m$ be a positive integer with $\operatorname{gcd}(N, m)=1$. We denote by $\mathbf{M}(N, m)$ any set of odd prime numbers relatively prime to $\mathrm{Nm}^{2}$ and congruent to $1 \bmod m$, such that $\mathbf{M}(N, m) \cap\left\{a+l c N m^{2} \mid l \in \mathbb{Z}\right\}$ is not empty whenever $a$ and $c$ are integers with $\operatorname{gcd}\left(c N m^{2}, a\right)=1$ and $a \equiv 1(\bmod m)$. (Such a set always exists by Dirichlet's theorem on primes in arithmetic progressions.)

TheOREM 1. Let $\left\{a_{n}\right\}_{n}$ and $\left\{b_{n}\right\}_{n}$ be two sequences in $\mathbb{C}$ with $a_{n}=$ $O\left(n^{\nu}\right)$ and $b_{n}=O\left(n^{\nu}\right)$ for some $\nu>0$. Define functions

$$
f(\tau)=\sum_{n=0}^{\infty} a_{n} e(n \tau) \quad \text { and } \quad g(\tau)=\sum_{n=0}^{\infty} b_{n} e(n \tau)
$$

on $\mathcal{H}$. Then statements $(\mathrm{A})$ and $(\mathrm{B})$ below are equivalent:

(A) $f(\tau)$ and $g(\tau)$ are in $\mathfrak{M}_{k}^{1}\left(\Gamma_{0}\left(N m^{2}\right) \cap \Gamma_{1}(m)\right)$ and satisfy $g(\tau)=$ $\left.f(\tau)\right|_{k}\left[w\left(N m^{2}\right)\right]$.

(B) The following conditions on the corresponding Dirichlet series hold. The series $\Lambda_{N m^{2}}(f ; s)+a_{0} / s+i^{k} b_{0} /(k-s)$ has a holomorphic continuation to the whole s-plane, is bounded on any vertical strip and satisfies

$$
\Lambda_{N m^{2}}(f ; s)=i^{k} \Lambda_{N m^{2}}(g ; k-s) .
$$

For any primitive Dirichlet character $\psi$ whose conductor $t$ is in $\mathbf{M}(N, m)$, the series $\Lambda_{N m^{2} t^{2}}(f, \psi ; s)$ has a holomorphic continuation to the whole s-plane, is bounded on any vertical strip and satisfies

$$
\Lambda_{N m^{2} t^{2}}(f, \psi ; s)=i^{k} \psi\left(m^{2}\right) C_{\psi} \Lambda_{N m^{2} t^{2}}(g, \bar{\psi} ; k-s) .
$$


3. Definitions and basic properties. Recall that the formal definition of second-order modular forms of level $N$ was given in the introduction. In the first result of this section we describe them in a slightly different way.

Lemma 3. A function $F: \mathcal{H} \rightarrow \mathbb{C}$ is in $\mathfrak{M}_{k}^{2}(N)$ if and only if

(a) $F(\tau)$ is holomorphic on $\mathcal{H}$,

(b) $F(\tau)$ has at most polynomial growth at the cusps,

(c) $\left.F(\tau)\right|_{k}\left[\gamma-I_{2}\right] \in \mathfrak{M}_{k}^{1}(N)$ for any $\gamma=\left(\begin{array}{cc}p & -v \\ -u N & q\end{array}\right)$ in $\Gamma_{0}(N)$ such that $u=0$ or whose entries $p, q$ are two distinct odd primes,

(d) $\left.F(\tau)\right|_{k}\left[\gamma-I_{2}\right]=0$ for every matrix $\gamma$ as above such that $u=0$ or $p+q \equiv \pm 2(\bmod u N)$.

Proof. Clearly the definition of second-order modular forms yields (a), (b) and (c). In fact, (c) holds for any matrix $\gamma$ in $\Gamma_{0}(N)$. As for (d), we observe that (iv) implies

$$
\left.F(\tau)\right|_{k}\left[ \pm\left(\begin{array}{ll}
1 & l \\
0 & 1
\end{array}\right)\right]=F(\tau)
$$

for all integers $l$. If $\gamma=\left(\begin{array}{cc}p & -v \\ -u N & q\end{array}\right)$ is in $\Gamma_{0}(N)$ with $u \neq 0$ and $p+q \equiv \pm 2$ $(\bmod u N)$, we can write $p+q= \pm 2+u N l$ for some $l$ in $\mathbb{Z}$. Then

$$
\gamma\left(\begin{array}{ll}
1 & l \\
0 & 1
\end{array}\right)=\left(\begin{array}{cc}
p & * \\
* & q-u N l
\end{array}\right)
$$

is a parabolic element of $\Gamma_{0}(N)$. Consequently, (iii) and (iv) yield

$$
\begin{aligned}
\left.F(\tau)\right|_{k}\left[\gamma-I_{2}\right] & =\left.\left.F(\tau)\right|_{k}\left[\gamma-I_{2}\right]\right|_{k}\left[\left(\begin{array}{ll}
1 & l \\
0 & 1
\end{array}\right)\right] \\
& =\left.\left.F(\tau)\right|_{k}\left[\gamma-I_{2}\right]\right|_{k}\left[\left(\begin{array}{ll}
1 & l \\
0 & 1
\end{array}\right)\right]+\left.F(\tau)\right|_{k}\left[\left(\begin{array}{ll}
1 & l \\
0 & 1
\end{array}\right)-I_{2}\right] \\
& =\left.F(\tau)\right|_{k}\left[\gamma\left(\begin{array}{ll}
1 & l \\
0 & 1
\end{array}\right)-I_{2}\right]=0 .
\end{aligned}
$$

Next we prove the equivalence in the other direction.

If $\gamma=\left(\begin{array}{cc}a & b \\ c N & d\end{array}\right) \in \Gamma_{0}(N)$ with $c \neq 0$ we consider the integral sequences $\{a+l c N \mid l \in \mathbb{Z}\}$ and $\{d+l c N \mid l \in \mathbb{Z}\}$. By Dirichlet's theorem on primes in arithmetic progressions, each of them contains infinitely many primes. In particular, there are arbitrarily large odd primes $p \neq q$ of the form $p=$ $a+t c N, q=d+s c N$ for some integers $t, s$. Put $u=-c$ and $v=-(b+s p+$ stuN $+q t)$. Then

$$
\widetilde{\gamma}=\left(\begin{array}{cc}
p & -v \\
-u N & q
\end{array}\right) \in \Gamma_{0}(N) \text { and } \gamma=\left(\begin{array}{cc}
1 & -t \\
0 & 1
\end{array}\right) \widetilde{\gamma}\left(\begin{array}{cc}
1 & -s \\
0 & 1
\end{array}\right) .
$$


Consequently, from (5) and hypothesis (c) one gets

$$
\begin{aligned}
& \left.F(\tau)\right|_{k}\left[\gamma-I_{2}\right] \\
= & \left.\left.F(\tau)\right|_{k}\left[\left(\begin{array}{cc}
1 & -t \\
0 & 1
\end{array}\right)-I_{2}\right]\right|_{k}\left[\widetilde{\gamma}\left(\begin{array}{cc}
1 & -s \\
0 & 1
\end{array}\right)\right]+\left.F(\tau)\right|_{k}\left[\widetilde{\gamma}\left(\begin{array}{cc}
1 & -s \\
0 & 1
\end{array}\right)-I_{2}\right] \\
= & \left.\left.F(\tau)\right|_{k}\left[\widetilde{\gamma}-I_{2}\right]\right|_{k}\left[\left(\begin{array}{cc}
1 & -s \\
0 & 1
\end{array}\right)\right]+\left.F(\tau)\right|_{k}\left[\left(\begin{array}{cc}
1 & -s \\
0 & 1
\end{array}\right)-I_{2}\right] \\
= & \left.F(\tau)\right|_{k}\left[\widetilde{\gamma}-I_{2}\right] .
\end{aligned}
$$

Thus $\left.F(\tau)\right|_{k}\left[\gamma-I_{2}\right]$ is in $\mathfrak{M}_{k}^{1}(N)$ and (iii) follows.

Suppose that the matrix $\gamma$ above is also parabolic. Then $a+d= \pm 2$. If $c=0$ there is nothing to prove. Otherwise $p+q=a+d+(t+s) c N \equiv \pm 2$ $(\bmod u N)$. Hence $(6)$ and $(\mathrm{d})$ yield $(\mathrm{iv})$.

We have already shown that $\left.F(\tau)\right|_{k}\left[\gamma-I_{2}\right] \in \mathfrak{M}_{k}^{1}(N)$ for all $\gamma \in \Gamma_{0}(N)$. Hence these functions must have polynomial growth at the cusps. As the same is true for $F(\tau)$, we obtain (ii).

Notice that our proof of (d) from the definition of second-order modular forms is valid for any matrix $\gamma=\left(\begin{array}{cc}a & b \\ c N & d\end{array}\right)$ in $\Gamma_{0}(N)$ with $a+d \equiv \pm 2(\bmod c N)$.

Consider next the following generalization of condition (d): For a holomorphic function $F: \mathcal{H} \rightarrow \mathbb{C}$, let

(D) $\left.F(\tau)\right|_{k}\left[\gamma-I_{2}\right]=0$ for every matrix $\gamma=\left(\begin{array}{cc}p & -v \\ -u N & q\end{array}\right)$ in $\Gamma_{0}(N)$ such that $u=0$ or $p+q \equiv \pm 2(\bmod u N)$ with $p$ an odd prime.

From equation (6) with $s=0$ one can show that (D) implies part (iv) in the definition of a second-order modular form.

4. Fourier series and Dirichlet characters. In this section we give a characterization of a second-order modular form using its Fourier series at infinity and twists of it by Dirichlet characters.

Throughout this section $F(\tau)$ and $G(\tau)$ are always holomorphic functions on $\mathcal{H}$ represented by the Fourier series $F(\tau)=\sum_{n=0}^{\infty} A_{n} e(n \tau)$ and $G(\tau)=$ $\sum_{n=0}^{\infty} B_{n} e(n \tau)$ with $A_{n}, B_{n}$ in $\mathbb{C}, A_{n}=O\left(n^{\nu}\right)$ and $B_{n}=O\left(n^{\nu}\right)$ for some $\nu>0$. Moreover they satisfy $G(\tau)=\left.F(\tau)\right|_{k}[w(N)]$.

Any $\gamma$ in $\Gamma_{0}(N)$ defines functions $f_{\gamma}, g_{\gamma}: \mathcal{H} \rightarrow \mathbb{C}$ by

$$
f_{\gamma}(\tau)=\left.F(\tau)\right|_{k}\left[\gamma-I_{2}\right] \quad \text { and } \quad g_{\gamma}(\tau)=\left.G(\tau)\right|_{k}\left[\gamma-I_{2}\right]
$$

For convenience we write $f_{p, v}(\tau)$ (resp. $g_{p, v}(\tau)$ ) instead of $f_{\gamma}(\tau)$ (resp. $\left.g_{\gamma}(\tau)\right)$ if $\gamma=\left(\begin{array}{cc}p & -v \\ -u N & q\end{array}\right) \in \Gamma_{0}(N)$. A straightforward computation shows that the notation is not ambiguous.

Let $f: \mathcal{H} \rightarrow \mathbb{C}$ be a holomorphic function represented by the series $f(\tau)=\sum_{n=0}^{\infty} a_{n} e(n \tau)$. We have already defined $f_{\chi}(\tau)$ for a primitive Dirichlet 
character $\chi$. It is also convenient to define $f_{\chi_{0}}(\tau)$ for the trivial character $\chi_{0} \bmod m$ whenever $m$ is a prime. Set $f_{\chi_{0}}(\tau)=f(\tau)-m \sum_{m \mid n} a_{n} e(n \tau)$.

One reason for such a definition is that $f_{\chi_{0}}(\tau)$ satisfies an identity like (2). Namely

$$
\left.\sum_{u(\bmod m)} f(\tau)\right|_{k}[\theta(u / m)]=m \sum_{m \mid n} a_{n} e(n \tau) .
$$

The analogy is more evident once we observe that $W\left(\chi_{0}\right)=-1$.

Lemma 4. Let $m$ be a positive integer with $\operatorname{gcd}(N, m)=1$. If $\chi$ is either a primitive Dirichlet character of conductor $m$ or the trivial Dirichlet character mod $m$ with $m$ prime, then

$$
\begin{aligned}
\left.G_{\chi}(\tau)\right|_{k}\left[w\left(N m^{2}\right)\right]- & C_{\chi} F_{\bar{\chi}}(\tau) \\
& =\left.C_{\chi} W(\chi)^{-1} \sum_{v(\bmod m)} \chi(v) f_{m, v}(\tau)\right|_{k}[\theta(v / m)] .
\end{aligned}
$$

Proof. Let $u, v$ be integers such that $-u v N \equiv 1(\bmod m)$. Then

$$
\theta(u / m) w\left(N m^{2}\right) \theta(-v / m)=m w(N) \gamma_{m, v}
$$

for some $\gamma_{m, v}=\left(\begin{array}{cc}m & -v \\ -u N & *\end{array}\right)$ in $\Gamma_{0}(N)$. If $\chi$ is a primitive (resp. trivial) character, we use (2) (resp. (7)) and (9) to get

$$
\begin{aligned}
\left.G_{\chi}(\tau)\right|_{k}[w & \left.\left(N m^{2}\right)\right]=\left.W(\bar{\chi})^{-1} \sum_{u(\bmod m)} \bar{\chi}(u) F(\tau)\right|_{k}\left[\gamma_{m, v} \theta(v / m)\right] \\
= & \left.W(\bar{\chi})^{-1} \chi(-N) \sum_{v(\bmod m)} \chi(v)\left\{F(\tau)+f_{m, v}(\tau)\right\}\right|_{k}[\theta(v / m)] \\
& =C_{\chi} F_{\bar{\chi}}(\tau)+\left.C_{\chi} W(\chi)^{-1} \sum_{v(\bmod m)} \chi(v) f_{m, v}(\tau)\right|_{k}[\theta(v / m)] .
\end{aligned}
$$

Notice that $f_{p, v}(\tau)$ is not defined if $\operatorname{gcd}(v, m)>1$, but then $\chi(v)=0$.

Definition 3. Let $m>1$ be an integer relatively prime to $N$. If $\chi$ is any primitive Dirichlet character of conductor $m$ or the trivial Dirichlet character $\bmod m$ with $m$ prime, let $H_{\chi}(\tau)$ be the left hand side of (8), i.e.

$$
H_{\chi}(\tau)=\left.G_{\chi}(\tau)\right|_{k}\left[w\left(N m^{2}\right)\right]-C_{\chi} F_{\bar{\chi}}(\tau)
$$

Let $p$ be a prime number with $\operatorname{gcd}(N, p)=1$. Then every Dirichlet character mod $p$ is either primitive or trivial. Hence equation (8) and the orthogonality relations for characters yield

$$
\left.(p-1) f_{p, v}(\tau)\right|_{k}[\theta(v / p)]=\sum_{\chi} \bar{\chi}(v) C_{\bar{\chi}} W(\chi) H_{\chi}(\tau)
$$

for any integer $v$ with $\operatorname{gcd}(v, p)=1$. Here the sum is over all characters $\bmod p$. 
Next we recall some standard notation and prove a technical lemma. Let $M$ and $m$ be two positive integers. As usual, the symbols $\Gamma_{0}^{0}(M, m)$ and $\Gamma^{1}(m)$ denote the groups

$$
\Gamma_{0}^{0}(M, m)=\left\{\left(\begin{array}{ll}
a & b \\
c & d
\end{array}\right) \in \mathrm{SL}_{2}(\mathbb{Z}) \mid c \equiv 0(\bmod M), b \equiv 0(\bmod m)\right\}
$$

and

$$
\Gamma^{1}(m)=\left\{\left(\begin{array}{ll}
a & b \\
c & d
\end{array}\right) \in \mathrm{SL}_{2}(\mathbb{Z}) \mid a \equiv 1(\bmod m), b \equiv 0(\bmod m)\right\} .
$$

Lemma 5. Let $p$ and $q$ be two distinct odd primes such that $p q-u v N=1$ for some $u, v$ in $\mathbb{Z}$. Then $\left\langle\Gamma_{0}\left(N p^{2}\right) \cap \Gamma_{1}(p), \Gamma_{0}^{0}\left(N, q^{2}\right) \cap \Gamma^{1}(q)\right\rangle$ (the subgroup of $\mathrm{SL}_{2}(\mathbb{Z})$ generated by the two intersections) is equal to $\Gamma_{0}(N)$.

Proof. Let $\left(\begin{array}{cc}a & b \\ c N & d\end{array}\right) \in \Gamma_{0}(N)$ with $a \equiv 1(\bmod q)$. There are integers $x, y$ such that

$$
\left(\begin{array}{cc}
x & y q^{2} \\
-c N & a
\end{array}\right) \in \Gamma_{0}^{0}\left(N, q^{2}\right) \cap \Gamma^{1}(q) .
$$

Thus

$$
\left(\begin{array}{cc}
x & y q^{2} \\
-c N & a
\end{array}\right)\left(\begin{array}{cc}
a & b \\
c N & d
\end{array}\right)=\left(\begin{array}{ll}
* & * \\
0 & 1
\end{array}\right)
$$

is in $\Gamma_{0}\left(N p^{2}\right) \cap \Gamma_{1}(p)$ as desired.

Let $\left(\begin{array}{cc}a & b \\ c N & d\end{array}\right) \in \Gamma_{0}(N)$ with $c$ relatively prime to $q$. There exists $c^{*} \in \mathbb{Z}$ such that $c c^{*} \equiv 1(\bmod q)$. Therefore

$$
\left(\begin{array}{cc}
1 & u v c^{*}(a-1) \\
0 & 1
\end{array}\right)\left(\begin{array}{cc}
a & b \\
c N & d
\end{array}\right)=\left(\begin{array}{cc}
a+u v c^{*}(a-1) c N & * \\
c N & d
\end{array}\right) \in \Gamma_{0}(N)
$$

with $a+u v c^{*}(a-1) c N \equiv 1(\bmod q)$. By the previous argument this matrix is in $\left\langle\Gamma_{0}\left(N p^{2}\right) \cap \Gamma_{1}(p), \Gamma_{0}^{0}\left(N, q^{2}\right) \cap \Gamma^{1}(q)\right\rangle$, and hence so is $\left(\begin{array}{cc}a & b \\ c N & d\end{array}\right)$.

Finally, we look at the case $\left(\begin{array}{cc}a & b \\ c N & d\end{array}\right) \in \Gamma_{0}(N)$ with $c \equiv 0(\bmod q)$. As $p \neq q$ there is $a^{*} \in \mathbb{Z}$ such that $a a^{*} \equiv 1(\bmod q)$ and $a^{*} \equiv 1(\bmod p N)$. Hence there are integers $d^{\prime}, b^{\prime}$ such that

$$
\left(\begin{array}{cc}
a^{*} & b^{\prime} \\
N p^{2} & d^{\prime}
\end{array}\right) \in \Gamma_{0}\left(N p^{2}\right) \cap \Gamma_{1}(p)
$$

and

$$
\left(\begin{array}{cc}
a^{*} & b^{\prime} \\
N p^{2} & d^{\prime}
\end{array}\right)\left(\begin{array}{cc}
a & b \\
c N & d
\end{array}\right)=\left(\begin{array}{cc}
a^{*} a+b^{\prime} c N & * \\
N p^{2} a+d^{\prime} c N & *
\end{array}\right) \in \Gamma_{0}(N)
$$

with $a^{*} a+b^{\prime} c N \equiv 1(\bmod q)$. By the previous argument this matrix, and therefore $\left(\begin{array}{cc}a & b \\ c N & d\end{array}\right)$, is in $\left\langle\Gamma_{0}\left(N p^{2}\right) \cap \Gamma_{1}(p), \Gamma_{0}^{0}\left(N, q^{2}\right) \cap \Gamma^{1}(q)\right\rangle$. 
The next proposition describes a second-order modular form in terms of the functions $H_{\chi}(\tau)$ given in (10). It is a key result for the proof of our main theorem.

Proposition 2. $F(\tau)$ is in $\mathfrak{M}_{k}^{2}(N)$ if and only if the following four statements hold:

$\left(\mathrm{a}^{\prime}\right) F(\tau)$ is holomorphic on $\mathcal{H}$.

$\left(\mathrm{b}^{\prime}\right) F(\tau)$ has at most polynomial growth at the cusps.

$\left(\mathrm{c}^{\prime}\right)$ For any odd prime $p$ relatively prime to $N$ and arbitrary Dirichlet character $\chi \bmod p$,

$$
H_{\chi}(\tau) \in \mathfrak{M}_{k}^{1}\left(\Gamma_{0}\left(N p^{2}\right) \cap \Gamma_{1}(p)\right) .
$$

$\left(\mathrm{d}^{\prime}\right)$ For any odd prime $p$ relatively prime to $N$ and arbitrary $u \in \mathbb{Z}$ with $(p \pm 1)^{2} \equiv 0(\bmod u N)$

$$
\sum_{\chi} \chi(u) C_{\bar{\chi}} W(\chi) H_{\chi}(\tau)=0
$$

where the sum is over all Dirichlet characters $\chi \bmod p$.

Proof. First we assume that $F(\tau)$ is in $\mathfrak{M}_{k}^{2}(N)$.

For any integer $v$ relatively prime to $p$ consider the matrix $\gamma_{p, v}$ in $\Gamma_{0}(N)$ determined by equation (9). Then $f_{p, v}(\tau)=\left.F(\tau)\right|_{k}\left[\gamma_{p, v}-I_{2}\right]$ is in $\mathfrak{M}_{k}^{1}(N)$ (see proof of Lemma 3) and therefore $\left.f_{p, v}(\tau)\right|_{k}[\theta(v / p)]$ is in $\mathfrak{M}_{k}^{1}\left(\Gamma_{0}\left(N p^{2}\right) \cap \Gamma_{1}(p)\right)$.

This fact and Lemma 4 yield $\left(\mathrm{c}^{\prime}\right)$.

In order to deduce $\left(\mathrm{d}^{\prime}\right)$ we write $(p \pm 1)^{2}=l u N$ for some $l$ in $\mathbb{Z}$. Then $l u N \equiv 1(\bmod p)$ and therefore $l \equiv-v(\bmod p)$ for any integer $v$ such that $-u v N \equiv 1(\bmod p)$. Put $l=-v+t p$ for some $t$ in $\mathbb{Z}$. Clearly

$$
(p \pm 1)^{2}=(t p-v) u N, \quad \text { i.e. } \quad p+\frac{1+v u N}{p}=\mp 2+t u N .
$$

Notice that $q=(1+v u N) / p \in \mathbb{Z},\left(\begin{array}{cc}p & -v \\ -u N & q\end{array}\right) \in \Gamma_{0}(N)$ and $p+q \equiv \pm 2$ $(\bmod u N)$. By Lemma 3 we conclude that $\left.f_{p, v}(\tau)\right|_{k}[\theta(v / p)]=0$. Now we use (11) and the congruence $-u v N \equiv 1(\bmod p)$ to get $\left(\mathrm{d}^{\prime}\right)$.

Next we prove the converse implication. Evidently, it suffices to show that $\left(\mathrm{a}^{\prime}\right),\left(\mathrm{b}^{\prime}\right),\left(\mathrm{c}^{\prime}\right)$ and $\left(\mathrm{d}^{\prime}\right)$ imply statements $(\mathrm{c})$ and $(\mathrm{d})$ of Lemma 3.

Let

$$
\gamma_{p, v_{0}}=\left(\begin{array}{cc}
p & -v_{0} \\
-u_{0} N & q
\end{array}\right) \in \Gamma_{0}(N)
$$

with $p, q$ two distinct odd primes. Then $\operatorname{gcd}(N, p)=\operatorname{gcd}(N, q)=1$ and $\operatorname{gcd}\left(v_{0}, p\right)=1\left(\right.$ resp. $\left.\operatorname{gcd}\left(u_{0}, q\right)=1\right)$. From $\left(\mathrm{c}^{\prime}\right)$ and $(11)$ we infer that $\left.f_{p, v_{0}}(\tau)\right|_{k}\left[\theta\left(v_{0} / p\right)\right]$, and hence $f_{p, v_{0}}(\tau)$, are in $\mathfrak{M}_{k}^{1}\left(\Gamma_{0}\left(N p^{2}\right) \cap \Gamma_{1}(p)\right)$. 
On the other hand, $w\left(N p^{2}\right)$ is in the normalizer of the group $\Gamma_{0}\left(N p^{2}\right) \cap$ $\Gamma_{1}(p), w\left(N p^{2}\right)^{2}=-N p^{2} I_{2}$ and $C_{\chi} C_{\bar{\chi}}=1$. Hence $\left(\mathrm{c}^{\prime}\right)$ also implies

$$
\left.H_{\chi}(\tau)\right|_{k}\left[w\left(N p^{2}\right)\right] \in \mathfrak{M}_{k}^{1}\left(\Gamma_{0}\left(N p^{2}\right) \cap \Gamma_{1}(p)\right)
$$

for all Dirichlet characters $\chi \bmod p$. Using the previous argument with $q$ (resp. $G(\tau)$ ) instead of $p($ resp. $F(\tau))$ we deduce that $g_{q,-u_{0}}(\tau) \in \mathfrak{M}_{k}^{1}\left(\Gamma_{0}\left(N q^{2}\right)\right.$ $\left.\cap \Gamma_{1}(q)\right)$. The identities $\left.f_{p, v_{0}}(\tau)\right|_{k}[w(N)]=g_{q,-u_{0}}(\tau)$ and

$$
w(N)^{-1}\left(\Gamma_{0}\left(N q^{2}\right) \cap \Gamma_{1}(q)\right) w(N)=\Gamma_{0}^{0}\left(N, q^{2}\right) \cap \Gamma^{1}(q)
$$

are easy to check. Thus

$$
f_{p, v_{0}}(\tau) \in \mathfrak{M}_{k}^{1}\left(\left\langle\Gamma_{0}\left(N p^{2}\right) \cap \Gamma_{1}(p), \Gamma_{0}^{0}\left(N, q^{2}\right) \cap \Gamma^{1}(q)\right\rangle\right) .
$$

By Lemma 5 we conclude that $f_{p, v_{0}}(\tau)$ is in $\mathfrak{M}_{k}^{1}(N)$, which is (c).

Finally, consider a matrix $\left(\begin{array}{cc}p & -v \\ -u N & q\end{array}\right)$ in $\Gamma_{0}(N)$ whose entries $p, q$ are distinct odd primes such that $p+q \equiv \pm 2(\bmod u N)$. Since $p q \equiv 1(\bmod u N)$ we have $p( \pm 2-p) \equiv 1(\bmod u N)$ and therefore $(p \mp 1)^{2} \equiv 0(\bmod u N)$. By hypothesis $\left(\mathrm{d}^{\prime}\right)$, equation $(11)$ and the relation $-u v N \equiv 1(\bmod p)$, we get $\left.f_{p, v}(\tau)\right|_{k}[\theta(v / p)]=0$ and therefore $f_{p, v}(\tau)=0$.

5. The main theorem. In this section we first state an auxiliary proposition and introduce a new kind of twist of Fourier series with Dirichlet characters. Then we restate our main result in terms of different twists of Dirichlet series and give the proof of it.

Proposition 3. Let $F_{1}(\tau)=\sum_{n=0}^{\infty} A_{n} e(n \tau), f_{1}(\tau)=\sum_{n=0}^{\infty} a_{n} e(n \tau)$, $F_{2}(\tau)=\sum_{n=0}^{\infty} B_{n} e(n \tau)$ and $f_{2}(\tau)=\sum_{n=0}^{\infty} b_{n} e(n \tau)$ be functions on $\mathcal{H}$ satisfying the hypothesis of Lemma 1 . Let $M$ be a positive integer. Then statements $\left(\mathrm{A}^{\prime}\right)$ and $\left(\mathrm{B}^{\prime}\right)$ below are equivalent.

$\left(\mathrm{A}^{\prime}\right) F_{1}(\tau)+f_{1}(\tau)=\left.F_{2}(\tau)\right|_{k}[\omega(M)]$ and $f_{1}(\tau)=\left.f_{2}(\tau)\right|_{k}[\omega(M)]$.

(B') $\Lambda_{M}\left(F_{1} ; s\right)$ and $\Lambda_{M}\left(F_{2} ; s\right)$ admit meromorphic continuations to the whole s-plane and they satisfy

$$
i^{k} \Lambda_{M}\left(F_{2} ; k-s\right)=\Lambda_{M}\left(F_{1} ; s\right)+\Lambda_{M}\left(f_{1} ; s\right)
$$

and

$$
\Lambda_{M}\left(f_{2} ; s\right)=i^{k} \Lambda_{M}\left(f_{1} ; k-s\right) .
$$

Moreover, both

$\Lambda_{M}\left(F_{1} ; s\right)+\frac{A_{0}}{s}+i^{k} \frac{B_{0}}{k-s}-i^{k} \frac{b_{0}}{k-s}$ and $\Lambda_{M}\left(f_{1} ; s\right)+\frac{a_{0}}{s}+i^{k} \frac{b_{0}}{k-s}$ are holomorphic on the whole complex s-plane and bounded on any vertical strip.

Proof. We can argue exactly as in the proof of Proposition 1. (See for example [10, pp. 119-121].) 
Definition 4. Let $f(\tau)=\sum_{n=0}^{\infty} a_{n} e(n \tau)$ and $g(\tau)=\sum_{n=0}^{\infty} b_{n} e(n \tau)$ be holomorphic functions on $\mathcal{H}$ satisfying the hypothesis of Lemma 1 and the relation $\left.f(\tau)\right|_{k}[w(N)]=g(\tau)$. Let $m$ be a positive integer with $\operatorname{gcd}(N, m)=1$ and $\chi$ a primitive Dirichlet character of conductor $m$ or the trivial character $\bmod m$ with $m$ prime. We define

$$
f^{\chi}(\tau)=\left.g_{\bar{\chi}}(\tau)\right|_{k}\left[w\left(N m^{2}\right)\right]
$$

The function $f^{\chi}(\tau)$ is similar to the twist of $f(\tau)$ by $\chi$. Compare for example the description of $f_{\chi}(\tau)$ in $(2)$ with the trivial identity

$$
f^{\chi}(\tau)=\left.W(\chi)^{-1} \sum_{u(\bmod m)} \chi(u) f(\tau)\right|_{k}\left[w(N) \theta(u / m) w\left(N m^{2}\right)\right] .
$$

In the particular case that $f(\tau)$ is a modular form in $\mathfrak{M}_{k}^{1}(N)$ one has $f^{\chi}(\tau)=$ $C_{\chi} f_{\chi}(\tau)$.

It is easy to see that the definition above and a straightforward application of Theorem 1 allow us to rephrase our main result (the Theorem in the introduction) as Theorem 2 below. In the latter form the similarities with Weil's converse theorem are more evident.

Theorem 2. Let $\left\{A_{n}\right\}_{n}$ and $\left\{B_{n}\right\}_{n}$ be two sequences in $\mathbb{C}$ with $A_{n}=$ $O\left(n^{\nu}\right)$ and $B_{n}=O\left(n^{\nu}\right)$ for some $\nu>0$. Define functions

$$
F(\tau)=\sum_{n=0}^{\infty} A_{n} e(n \tau) \quad \text { and } \quad G(\tau)=\sum_{n=0}^{\infty} B_{n} e(n \tau)
$$

on $\mathcal{H}$. Then statements $(\mathrm{A})$ and $(\mathrm{B})$ below are equivalent.

(A) $F(\tau)$ and $G(\tau)$ are in $\mathfrak{M}_{k}^{2}(N)$ with $G(\tau)=\left.F(\tau)\right|_{k}[w(N)]$.

(B) The following conditions on the corresponding Dirichlet series hold. The series $\Lambda_{N}(F ; s)+A_{0} / s+i^{k} B_{0} /(k-s)$ has a holomorphic continuation to the whole s-plane, is bounded on any vertical strip and satisfies

$$
\Lambda_{N}(F ; s)=i^{k} \Lambda_{N}(G ; k-s) .
$$

If $a_{0}^{\bar{\chi}}\left(\right.$ resp. $\left.b_{0}^{\chi}\right)$ is the constant term in the Fourier series of $F^{\bar{\chi}}(\tau)$ $\left(\right.$ resp. $\left.G^{\chi}(\tau)\right)$, where $\chi$ is any Dirichlet character $\bmod p$ and $p$ is an odd prime with $\operatorname{gcd}(N, p)=1$, then:

(B.1) The function

$$
\Lambda_{N p^{2}}\left(F^{\bar{\chi}} ; s\right)-C_{\chi} \Lambda_{N p^{2}}\left(F_{\bar{\chi}} ; s\right)+\frac{a_{0}^{\bar{\chi}}}{s}-i^{k} C_{\chi} \frac{b_{0}^{\chi}}{k-s}
$$

has a holomorphic continuation to the whole s-plane bounded on any vertical strip.

(B.2) For any primitive Dirichlet character $\psi$ of conductor $t$ in $\mathbf{M}(N, p)$, the function $\Lambda_{N p^{2} t^{2}}\left(F^{\bar{\chi}}, \psi ; s\right)-C_{\chi} \Lambda_{N p^{2} t^{2}}\left(F_{\bar{\chi}}, \psi ; s\right)$ 
has a holomorphic continuation to the whole s-plane, and is bounded on any vertical strip. Moreover

$$
\begin{aligned}
& \Lambda_{N p^{2} t^{2}}\left(F^{\bar{\chi}}, \psi ; s\right)-C_{\chi} \Lambda_{N p^{2} t^{2}}\left(F_{\bar{\chi}}, \psi ; s\right) \\
& =i^{k} \psi\left(p^{2}\right) C_{\psi} \Lambda_{N p^{2} t^{2}}\left(G_{\chi}, \bar{\psi} ; k-s\right) \\
& \quad-i^{k} \psi\left(p^{2}\right) C_{\psi} C_{\chi} \Lambda_{N p^{2} t^{2}}\left(G^{\chi}, \bar{\psi} ; k-s\right) .
\end{aligned}
$$

(B.3) The Dirichlet series $\sum_{\chi} \chi(u) C_{\bar{\chi}} W(\chi) \Lambda_{N p^{2}}\left(G_{\chi} ; s\right)$ (where the sum is over all Dirichlet characters $\bmod p)$ has a holomorphic continuation to the whole s-plane, is bounded on vertical strips and satisfies

$$
\begin{aligned}
& \sum_{\chi} \chi(u) C_{\bar{\chi}} W(\chi)\left\{i^{k} \Lambda_{N p^{2}}\left(G_{\chi} ; k-s\right)-C_{\chi} \Lambda_{N p^{2}}\left(F_{\bar{\chi}} ; s\right)\right\}=0 \\
& \text { whenever } u \in \mathbb{Z} \text { is such that }(p \pm 1)^{2} \equiv 0(\bmod u N) .
\end{aligned}
$$

Proof. Assume that $F(\tau)$ is in $\mathfrak{M}_{k}^{2}(N)$ and $G(\tau)=\left.F(\tau)\right|_{k}[w(N)]$. Then $F(\tau)$ and $G(\tau)$ satisfy the hypothesis of Lemma 1 and by Proposition 3 we get the first part of (B), i.e. the function $\Lambda_{N}(F ; s)+A_{0} / s+i^{k} B_{0} /(k-s)$ has a holomorphic continuation to the whole $s$-plane, is bounded on any vertical strip and satisfies (13).

Now let $\chi$ be a character as in the theorem. Proposition 2 shows that

$$
H_{\chi}(\tau)=F^{\bar{\chi}}(\tau)-C_{\chi} F_{\bar{\chi}}(\tau) \quad \text { is in } \mathfrak{M}_{k}^{1}\left(\Gamma_{0}\left(N p^{2}\right) \cap \Gamma_{1}(p)\right) .
$$

Hence Theorem 1 implies that $\Lambda_{N p^{2}}\left(F^{\bar{\chi}} ; s\right)-C_{\chi} \Lambda_{N p^{2}}\left(F_{\bar{\chi}} ; s\right)+a_{0}^{\bar{\chi}} / s-$ $i^{k} C_{\chi} b_{0}^{\chi} /(k-s)$ has holomorphic continuation to the whole $s$-plane bounded on any vertical strip.

We also use Theorem 1 to conclude that for any primitive Dirichlet character $\psi$ as above the series $\Lambda_{N p^{2} t^{2}}\left(F^{\bar{\chi}}-C_{\chi} F_{\bar{\chi}}, \psi ; s\right)$ has a holomorphic continuation to the whole $s$-plane, is bounded on any vertical strip and satisfies

$$
\Lambda_{N p^{2} t^{2}}\left(F^{\bar{\chi}}-C_{\chi} F_{\bar{\chi}}, \psi ; s\right)=i^{k} \psi\left(p^{2}\right) C_{\psi} \Lambda_{N p^{2} t^{2}}\left(G_{\chi}-C_{\chi} G^{\chi}, \bar{\psi} ; k-s\right) .
$$

From these equations and the linearity of the $L$-function we get (14).

For the proof of (B.3) consider

$$
\mathcal{F}_{p, u}(\tau)=\sum_{\chi} \chi(u) C_{\bar{\chi}} W(\chi) G_{\chi}(\tau) \quad \text { and } \quad \mathcal{G}_{p, u}(\tau)=\sum_{\chi} \chi(u) W(\chi) F_{\bar{\chi}}(\tau),
$$

where the sums are over all Dirichlet characters $\bmod p$ and $u$ is any integer such that $(p \pm 1)^{2} \equiv 0(\bmod u N)$. Since the functions defined by the Fourier series $F_{\bar{\chi}}(\tau)$ and $G_{\chi}(\tau)$ satisfy the hypothesis of Lemma 1, the same is true for $\mathcal{F}_{p, u}(\tau)$ and $\mathcal{G}_{p, u}(\tau)$. 
Part $\left(\mathrm{d}^{\prime}\right)$ of Proposition 2 is equivalent to $\left.\mathcal{F}_{p, u}(\tau)\right|_{k}\left[w\left(N p^{2}\right)\right]=\mathcal{G}_{p, u}(\tau)$, and this identity yields (B.3) by Proposition 1.

For the converse implication we argue as follows. The growth of the Fourier coefficients $A_{n}, B_{n}$, together with Lemma 2, implies that both series $F(\tau)$ and $G(\tau)$ define holomorphic functions on $\mathcal{H}$. From the particular meromorphic continuation of $\Lambda_{N}(F ; s)$ to the $s$-plane, equation (13) and Proposition 1 we get $G(\tau)=\left.F(\tau)\right|_{k}[w(N)]$.

Next we consider any odd prime $p$ with $\operatorname{gcd}(N, p)=1$ and any Dirichlet character $\chi \bmod p$.

Claim. The function $H_{\chi}(\tau)$ is invariant under the translation $\tau \mapsto \tau+1$.

Proof of the Claim. Let $\mathcal{F}_{p, u}(\tau)$ and $\mathcal{G}_{p, u}(\tau)$ be as above. We use Proposition 1 to deduce from (B.3) that

$$
\mathcal{G}_{p, u}(\tau)=\left.\mathcal{F}_{p, u}(\tau)\right|_{k}\left[w\left(N p^{2}\right)\right] .
$$

Notice that (16) is equivalent to the equation in part $\left(\mathrm{d}^{\prime}\right)$ of Proposition 2. Arguing as in the proof of the latter we can deduce from (16) the invariance of $F(\tau)$ under $\left(\begin{array}{cc}p & -v \\ -u N & q\end{array}\right)$ in $\Gamma_{0}(N)$ where $q$ is any integer with $p+q \equiv \pm 2$ $(\bmod u N)$. Now we recall the remark after Lemma 3 and conclude that $F(\tau)$ is invariant under any parabolic element of $\Gamma_{0}(N)$. Since the conjugate of any parabolic matrix by $w(N)$ is parabolic, $G(\tau)=\left.F(\tau)\right|_{k}[w(N)]$ is also invariant under any parabolic element of $\Gamma_{0}(N)$. This fact and the identities

$$
w\left(N p^{2}\right)\left(\begin{array}{ll}
1 & 1 \\
0 & 1
\end{array}\right)=\left(\begin{array}{cc}
1 & 0 \\
-N p^{2} & 1
\end{array}\right) w\left(N p^{2}\right)
$$

and

$$
\theta\left(\frac{u}{p}\right)\left(\begin{array}{cc}
1 & 0 \\
-N p^{2} & 1
\end{array}\right)=\left(\begin{array}{cc}
1-u N p & u^{2} N \\
-N p^{2} & 1+u N p
\end{array}\right) \theta\left(\frac{u}{p}\right)
$$

imply that $F^{\bar{\chi}}(\tau)=\left.G_{\chi}(\tau)\right|_{k}\left[w\left(N p^{2}\right)\right]$ is invariant under the translation $\tau \mapsto \tau+1$. This implies the Claim.

Consequently, $H_{\chi}(\tau)=\sum_{n \in \mathbb{Z}} a_{n} e(n \tau)$ for some $a_{n} \in \mathbb{C}$. Using now the estimate $G_{\chi}(\tau)=O\left(\operatorname{Im}(\tau)^{-\nu-1}\right)$ as $\operatorname{Im}(\tau) \rightarrow 0$ we conclude that $H_{\chi}(\tau)=$ $\sum_{n \geq 0} a_{n} e(n \tau)$.

Similarly one has $\left.H_{\chi}(\tau)\right|_{k}\left[w\left(N p^{2}\right)\right]=\sum_{n \geq 0} b_{n} e(n \tau)$ for some coefficients $b_{n}$ in $\mathbb{C}$. Proposition 3 then implies

$$
\Lambda_{N p^{2}}\left(H_{\chi} ; s\right)=i^{k} \Lambda_{N p^{2}}\left(G_{\chi} ; k-s\right)-C_{\chi} \Lambda_{N p^{2}}\left(F_{\bar{\chi}} ; s\right) .
$$

Notice that the linearity of the $L$-function yields 


$$
\Lambda_{N p^{2} t^{2}}\left(H_{\chi}, \psi ; s\right)=\Lambda_{N p^{2} t^{2}}\left(F^{\bar{\chi}}, \psi ; s\right)-C_{\chi} \Lambda_{N p^{2} t^{2}}\left(F_{\bar{\chi}}, \psi ; s\right)
$$

and

$$
\Lambda_{N p^{2} t^{2}}\left(\left.H_{\chi}\right|_{k}\left[w\left(N p^{2}\right)\right], \psi ; s\right)=\Lambda_{N p^{2} t^{2}}\left(G_{\chi}, \psi ; s\right)-C_{\chi} \Lambda_{N p^{2} t^{2}}\left(G^{\chi}, \psi ; s\right) .
$$

If we now use the hypothesis in (B.2) and Theorem 1 we conclude that $H_{\chi}(\tau)$ is in $\mathfrak{M}_{k}^{1}\left(\Gamma_{0}\left(N p^{2}\right) \cap \Gamma_{1}(p)\right)$.

We already know that $\left.F(\tau)\right|_{k}\left[\gamma-I_{2}\right]=0$ for any parabolic $\gamma$ in $\Gamma_{0}(N)$. This identity and the estimate $F(\tau)=O\left(\operatorname{Im}(\tau)^{-\nu-1}\right)$ as $\operatorname{Im}(\tau) \rightarrow 0$ (see Lemma 2) imply that $F(\tau)$ has at most polynomial growth at every cusp of $\Gamma_{0}(N)$ (details as in [10, p. 41] for example).

By Proposition 2 we conclude that $F(\tau)$ is in $\mathfrak{M}_{k}^{2}(N)$.

Finally, we want to point out that it is possible to refine our converse Theorem 2 to a statement where only finitely many twists of $F(\tau)$ and $G(\tau)$ are necessary. One can proceed as in [14] where Razar makes such a refinement of Weil's theorem.

\section{References}

[1] G. Chinta, N. Diamantis and C. O'Sullivan, Second order modular forms, Acta Arith. 103 (2002), 209-223.

[2] G. Chinta and D. Goldfeld, Grössencharakter L-functions of real quadratic fields twisted by modular symbols, Invent. Math. 144 (2001), 435-449.

[3] N. Diamantis, M. Knopp, G. Mason and C. O'Sullivan, L-functions of second order cusp forms, preprint.

[4] N. Diamantis and C. O'Sullivan, Hecke theory of series formed with modular symbols and relations among convolution L-functions, Math. Ann. 318 (2000), 85-105.

[5] - - - The dimensions of spaces of holomorphic second order automorphic forms and their cohomology, preprint, arXiv:math.NT/0411135.

[6] D. Goldfeld, Zeta functions formed with modular symbols, in: Proc. Sympos. Pure Math. 66, Amer. Math. Soc., 1999, 111-121.

[7] D. Goldfeld and E. Gunnells, Eisenstein series twisted by modular symbols for the group $\mathrm{GL}_{n}$, Math. Res. Lett. 7 (2000), 747-756.

[8] J. Jorgenson and C. O'Sullivan, Convolution Dirichlet series and a Kronecker limit formula for second-order Eisenstein series, Nagoya Math. J. 179 (2005), 47-102.

[9] P. Kleban and D. Zagier, Crossing probabilities and modular forms, J. Statist. Phys. 113 (2003), 431-454.

[10] T. Miyake, Modular Forms, Springer, Berlin, 1989.

[11] C. O'Sullivan, Properties of Eisenstein series with modular symbols, J. Reine Angew. Math. 518 (2000), 163-186.

[12] Y. Petridis, Spectral deformations and Eisenstein series associated with modular symbols, Int. Math. Res. Not. 2002, no. 19, 991-1006.

[13] Y. Petridis and M. S. Risager, Modular symbols have a normal distribution, Geom. Funct. Anal. 14 (2004), 1013-1043. 
[14] M. Razar, Modular forms for $\Gamma_{0}(N)$ and Dirichlet series, Trans. Amer. Math. Soc. 231 (1997), 489-495.

[15] A. Weil, Über die Bestimmung Dirichletscher Reihen durch Funktionalgleichungen, Math. Ann. 168 (1967), 149-156.

Department of Mathematics

ETH Zürich

CH-8096 Zürich, Switzerland

E-mail: ozlem@math.ethz.ch
Departamento de Matemáticas

Facultad de Ciencias

Universidad de Chile

Casilla 653, Santiago, Chile

E-mail: ymartin@uchile.cl

Received on 30.5.2005

and in revised form on 10.3.2006 